\title{
ВMJ Global Health Building caregivers' emotional, parental and social support skills to prevent violence against adolescent girls: findings from a cluster randomised controlled trial in Democratic Republic of Congo
}

To cite: Stark L, Seff I, Asghar K et al. Building caregivers' emotional, parental and social support skills to prevent violence against adolescent girls: findings from a cluster randomised controlled trial in Democratic Republic of Congo. BMJ Glob Health 2018;3:e000824. doi:10.1136/ bmjgh-2018-000824

Handling editor Seye Abimbola

- Additional material is published online only. To view please visit the journal online (http://dx.doi.org/10.1136/ bmjgh-2018-000824).

Received 13 March 2018 Revised 12 June 2018 Accepted 16 June 2018

\section{Linked}

- https://doi.org/10.1136/ bmjgh-2018-000825

Check for updates

(C) Author(s) (or their employer(s)) 2018. Re-use permitted under CC BY-NC. No commercial re-use. See rights and permissions. Published by BMJ.

For numbered affiliations see end of article.

Correspondence to Dr Lindsay Stark; Is2302@cumc.columbia.edu

\section{ABSTRACT}

Introduction Parenting programmes are increasingly popular for reducing children's exposure to interpersonal violence in low/middle-income countries, but there is limited evidence on their effectiveness. We investigated the incremental impact of adding a caregiver component to a life skills programme for adolescent girls, assessing girls' exposure to violence (sexual and others) and caregivers' gender attitudes and parenting behaviours.

Methods In this two-arm, single-blinded, cluster randomised controlled trial, we recruited 869 adolescent girls aged 10-14 and 764 caregivers in South Kivu, Democratic Republic of Congo. Following a baseline survey, participants were divided into 35 clusters based on age, language and location. Eighteen clusters were randomised to the treatment arm and 17 clusters to the wait-list control arm. Adolescent girls in both arms received 32 life skills sessions; caregivers in the treatment arm received 13 complementary caregiver sessions. The primary outcome was girls' self-reported exposure to sexual violence in the last 12 months; secondary outcomes included self-reports of specific forms of sexual violence, physical and emotional violence, transactional sex, child marriage for girls and parenting behaviours for caregivers. Intent-to-treat and per-protocol analyses were conducted. Results At 12 months of follow-up, the intervention showed no impact on sexual violence (adjusted $\mathrm{OR}=0.95 ; 95 \% \mathrm{Cl} 0.65$ to 1.37) or any secondary outcomes for girls. The intervention was associated with improved supportive parenting behaviours. Protocol adherence was also associated with improvements in these outcomes.

Conclusion While the caregiver curriculum improved some parenting outcomes, additional programmatic adaptations may be needed to reduce adolescent girls' violence exposure in humanitarian settings.

Trial registration number NCT02384642.

\section{INTRODUCTION}

Parenting programmes' proven success in reducing violence exposure for children in

\section{Key questions}

What is already known?

- Caregivers who participate in parenting programmes exhibit healthier child-parent relationships, but it is unknown whether integrating contents on adolescent sexual risks can impact sexual violence exposure for adolescents, particularly in humanitarian settings.

What are the new findings?

- Our study found a caregiver programme to have positive impacts on caregivers' parenting styles but no evidence of reduced girls' exposure to sexual violence.

What do the new findings imply?

- The findings imply that further research is needed to ascertain whether parenting programmes can reduce risk of sexual violence for adolescent girls.

- In particular, future research should assess the impact of programmes that are modified to include more frequent sessions and wider inclusion of male caregivers and other influential members in adolescent girls' lives.

high-income countries has led to their emergent utilisation in low/middle-income countries (LMIC) in recent years. ${ }^{1}$ Employing a structured curriculum that stresses the importance of open communication, positive parentchild relationships and non-violent disciplinary methods, these interventions aim to foster parenting behaviours that allow children to thrive and reduce their exposure to violence. ${ }^{2}$ Nascent evidence from LMIC and humanitarian contexts demonstrates that caregivers who participate in these interventions exhibit healthier child-parent relationships and are less 
likely to use harsh or corporal punishment. ${ }^{3-6}$ Parenting programmes that integrate content on sexual risks specific to children and adolescents may also usefully increase parents' awareness of these issues and empower them to support their children as they move into adolescence. ${ }^{27}$

Notably, the evidence base for parenting programmes in LMIC and humanitarian settings almost exclusively targets outcomes for early and middle childhood. ${ }^{8}$ Little is known about how these programmes might similarly transform the lives of adolescents, and adolescent girls in particular. ${ }^{9}{ }^{10}$ Owing to the intersectionality of age and gender, adolescent girls are uniquely vulnerable to multiple forms of violence including sexual violence, intimate partner violence (IPV), family violence, corporal punishment and child marriage. ${ }^{11-13}$ As caregivers play an instrumental role in adolescent girls' lives, the types of emotional, parental and social support they provide may plausibly shape girls' susceptibility to violence through a number of pathways. ${ }^{14}{ }^{15}$ For example, caregiver communication and connectedness can reduce adolescents' risk of IPV exposure. ${ }^{16}{ }^{17}$ Conversely, caregiver use of corporal punishment is associated with adolescent exposure to peer-perpetrated violence. ${ }^{18}$ Parents who favour corporal punishment over non-violent discipline are themselves frequent perpetrators of violence against adolescent girls. ${ }^{13}$ Additionally, the well-established association between exposure to family violence in young adolescence and later IPV exposure suggests that engaging caregivers to reinforce non-violent parental behaviours might contribute to declines in multiple types of violence for adolescent girls. ${ }^{19}$

Further, acceptance of IPV is deeply entrenched in social norms characterised by male-dominated decision-making, masculine ideologies that inherently encourage male aggression and gender roles that devalue women. ${ }^{20-22}$ In communities where gender inequity is normative, both men and women are more likely to display attitudinal acceptance of IPV. ${ }^{23}$ Caregivers who subscribe to these norms may inculcate girls with similar gender inequitable ideologies, further perpetuating cycles of violence.

Understanding how engaging caregivers as allies in building adolescent life skills and empowerment might mitigate violence exposure for adolescent girls is especially critical in conflict-affected settings, where the threat of violence is a pervasive one. ${ }^{24}$ Evidence suggests that conflicts can impact parents' stress, mental health and resilience in ways that may compromise positive parenting and increase child maltreatment. ${ }^{25}{ }^{26}$ Parents may also marry off their adolescent girls in an attempt to 'protect' them from sexual violence or to reduce economic strain during times of crisis. ${ }^{27}$ Further, gendered vulnerabilities combined with the financial instability frequently experienced in humanitarian settings increase adolescent girls' likelihood of experiencing sexual exploitation. ${ }^{28}$ The salience of child marriage and gender inequitable power dynamics in these settings leave girls vulnerable to coercion, especially as it relates to sexual activity. ${ }^{29}{ }^{30}$ Lastly, in protracted humanitarian settings, family support may be an important buffer against violence for adolescent girls as reliability of other resources, such as livelihood opportunities, becomes more volatile. $^{31}$

In the Democratic Republic of Congo (DRC), the setting for this study, more than half of ever-partnered girls aged 15-19 years reported experiencing physical or sexual IPV in their lifetime; and nearly $20 \%$ of sexually active 15-19 year-olds in the Central and West African regions reported their sexual debut as forced.$^{32}$ Baseline data from the present study show that even younger girls in the DRC face substantial risks of sexual violence, with $26.5 \%$ of girls aged 10-14 reporting having experienced any form of sexual violence in the last 12 months. While sexual violence has been a primary focus of research on women and girls in the DRC, there are indications of other forms of violence and trauma; for example, slightly less than $60 \%$ of girls aged 13-21 reported witnessed someone being killed in the Congo. ${ }^{33}$ Indeed, baseline findings from the present study show over $30 \%$ prevalence of physical and emotional violence exposure among girls aged $10-14 .{ }^{11}$ Research shows that girls who experience IPV (physical and sexual) during adolescence are more likely to develop depression, post-traumatic stress disorder, suicide ideation and sexual and reproductive health issues. ${ }^{34-36}$ Evidence also suggests that exposure to violence in adolescence, as compared with other stages of the life course, has a negative impact on the subsequent trajectory of development and well-being, extending well into adulthood. ${ }^{37-39}$

To add to the evidence base on parenting programmes and violence outcomes for adolescent girls in humanitarian contexts, Columbia University and the International Rescue Committee (IRC) evaluated the effectiveness of an adolescent girl's life skills programme, Creating Opportunities through Mentorship, Parental Involvement, and Safe Spaces (COMPASS), in three countries. In DRC, incremental effectiveness of the COMPASS caregiver curriculum was compared with receiving the girls' curriculum only. This article presents results from a cluster randomised controlled trial (RCT) to assess the added impact of the COMPASS caregiver curriculum to the girls' curriculum on adolescent girls' exposure to sexual and other forms of violence, and caregivers' gender attitudes and parenting behaviours, compared with the COMPASS girls' programming alone.

\section{METHODS}

\section{Study design and sample}

COMPASS is a programme implemented with refugees living in camps on the Sudan/Ethiopia border, conflict-affected communities in eastern DRC and displaced populations in north-east Pakistan. This multicountry programme offered a unique opportunity to evaluate the overall effectiveness of the approach, and whether certain components of the intervention meaningfully contributed to reducing violence against adolescent girls. Columbia University evaluated COMPASS in all three locations, each of which had a 
different research objective and design. In Ethiopia, the impact evaluation studied whether the core adolescent and caregiver programme components had an impact on girls' experiences of violence and social outcomes. The Ethiopia evaluation found that the intervention had a positive impact on measures of social support and attitudes around rites of passage, but had no effect on exposure to violence. ${ }^{40}$ Given that parents and caregivers can be both perpetrators of violence against girls as well as sources of protection, the DRC evaluation sought to provide important evidence around the value of the additional investment in parents and caregivers to prevent or reduce violence against girls. Specifically, the DRC study measured the incremental impact of the caregiver curriculum, as compared with the adolescent programme alone, on girls' experiences of violence and social outcomes, along with attitudes and characteristics of participating caregivers (see online supplementary file 1-COMPASS DRC IRB protocol). In Pakistan, the evaluation assessed the acceptability of the programme to adolescent girls and parents/caregivers in their context, and measured changes in girls' social and health outcomes over the course of the programme. This article presents findings from the evaluation implemented in DRC.

This two-arm, wait-list, cluster RCT took place across 14 sites in South Kivu, DRC, from July 2015 to October 2016. Civilians in eastern DRC, including the provinces of North Kivu and South Kivu, have been affected by conflict and uncertainty for almost 20 years. All study sites included predominantly rural villages of varied size and primarily hosted stable populations, including recent returnees. IRC staff introduced the COMPASS programme to selected communities, and adolescent girls aged 10-14 years and a caregiver of their choosing were invited to enrol. Following programme enrolment, girls were invited to participate in the study. After obtaining written consent from caregivers for all participating girls, girls were asked for assent to participate. For limited-literacy participants, interviewers read informed consent documents aloud. Caregivers participating in the study were also asked to provide written informed consent for their own participation. Caregivers were not required to participate for adolescent girls to be eligible for the study.

Study participants were then divided into clusters based on age (10-12 and 13-14 years old), language (Mashi and Swahili) and location. Clusters also served as programme groups; girls attended COMPASS sessions with others in their cluster only. Using uniform distribution in $\mathrm{R}$, clusters were then randomised by Columbia University to receive either: (1) Adolescent Girl Safe Spaces and life skills programming (AGSS) (wait-list control), or (2) AGSS and Caregiver Discussion Groups (CDG) (treatment). In the case that two or more siblings were enrolled in the study, they were randomised to the same treatment condition to minimise contamination, and linked to the same caregiver. Given the nature of the COMPASS intervention, treatment assignments were non-blinded for participants. Finally, participants were not compensated for their involvement in the study.

\section{Intervention}

COMPASS programming for adolescent girls and caregivers is composed of a combination of approaches, including the provision of safe spaces, building life skills and social assets, engaging girls in relationships with mentors and engaging caregivers as support systems and advocates for girls. These strategies have been shown in other contexts to successfully increase girls' feelings of safety, social support systems and aspirations to delay marriage. ${ }^{40}$ It was theorised that this combination of activities would increase girls' human, social, physical and financial assets to protect themselves from violence and respond to threats or incidents of violence; improve attitudes, knowledge and skills of influential people in girls' lives to protect girls from violence and support girls to be safe from violence; and increase capacity of service providers to provide safe, girl-friendly and life-saving services. ${ }^{41}$

Girls were grouped into units of approximately 20-25 participants and each group was paired with a female mentor aged 18-30. All female mentors were identified within the community by adolescent girls themselves and received training from the IRC. Criteria for serving as a female mentor included being able to read and write, having a basic knowledge of French, possessing positive attitudes towards adolescent girls and being available to facilitate sessions at least once a week. In addition to training mentors, IRC staff provided regular coaching, and attended and supported the delivery of early sessions in each community. Groups met with their mentor once a week, for 32 weeks, in spaces belonging to local community-based organisations. A different topic was discussed in each session, including interpersonal disagreement and resolution, decision-making, gender equitable norms, reproductive health and safety planning, among others (further details on the COMPASS curriculum for girls can be found in Stark and Asghar, forthcoming). ${ }^{40}$ In addition to exposing girls to the COMPASS curriculum, the sessions provided an opportunity for girls to develop social networks with peers, strengthen a healthy mentee-mentor relationship and build confidence. Girls in both the treatment and wait-list control arms received life skills programming.

Caregivers assigned to the treatment arm of the study participated in similarly structured caregiver discussion groups. Unlike the girls' sessions, caregivers received 13 sessions held on average once per month. The objective of these discussion groups was to create an open environment for parents and other primary caregivers to discuss their experiences and difficulties raising adolescent girls, and to foster caregiver understanding and support for girls' health, well-being and empowerment. Sessions were facilitated by IRC staff who delivered a structured curriculum including content on supporting adolescent girls, positive caregiver-girl relationships, fostering 
gender equitable attitudes and non-violent methods of child discipline. Additionally, information on development and cultural issues specific to adolescent girls was covered. All sessions were taught using a combination of didactic lessons, facilitated group discussion and skills practice. The caregiver curriculum followed the adolescent curriculum so as to be mutually supportive and ensure the content being discussed with the adolescent girls was understood and reiterated by caregivers (see online supplementary appendix A for a description of the caregiver group structure and curriculum). In addition to the core content for each session, the curriculum manual included guidance for facilitators including objectives and discussion questions for each session, guidance on teaching and practising positive caregiving skills, facilitation tips and best practices, language and messaging, the impact of group dynamics, and problem solving and crisis management. All IRC facilitators were experienced Women's Protection and Equality team members with background in gender-based violence prevention and response programming. Each mentor or IRC staff member was assigned to either one girl group or caregiver group, respectively; no group leader worked with more than one programme group.

\section{Data collection}

Baseline surveys were administered to 869 girls and 764 caregivers from May to July 2015, and endline data were collected after intervention from August to October 2016. Survey data collectors were trained by Columbia University and IRC. Both the girls' and caregivers' surveys were translated into French, Swahili and Mashi, and interviewers and questionnaires were appropriately matched to respondents by language. All data were collected in empty, private rooms in local community-based organisations. Two methods of data collection were employed for the girls' survey. Less sensitive questions from the questionnaire were administered through face-to-face interviews using Computer Assisted Personal Interviewing (CAPI). More sensitive questions, such as those on violence exposure and sexual behaviours, were self-administered by girls using Audio Computer Assisted Self-Interviewing (ACASI) ${ }^{42}$ Additionally, some of the violence and sexual health questions were not administered to girls aged 10-12 years for ethical and cultural reasons. All caregiver surveys were administered using CAPI. Primary and secondary outcomes for girls and caregivers can be found in table 1. All girl and caregiver outcomes were self-reported by girls and caregivers, respectively.

\section{Analysis}

Frequencies, ranges, means and SDs were examined for all outcomes and demographic characteristics (see online supplementary file 2-COMPASS DRC: statistical analysis plan). Pearson $\mathrm{X}^{2}$ tests and t-tests were used to examine the independence of dichotomous and continuous baseline characteristics, respectively, between treatment arms, protocol adherence groups and those who did and did not complete the endline survey. To assess the effects of the intervention, both intent-to-treat (ITT) and per-protocol (PP) analyses were implemented. PP analysis was restricted to girls who attended at least $75 \%$ of sessions and examined the effect of an adolescent girl's caregiver attending at least $75 \%$ of programme sessions. Additionally, as caregivers may have had more than one girl participate in the programme, we also restricted assessment of attendance protocol data to the oldest daughter in each family. PP analysis for caregiver outcomes thus assessed differences in outcomes across three groups: caregivers in the wait-list control arm; those in the treatment arm who attended less than $75 \%$ of sessions (non-PP adherence); and those in the treatment arm who attended at least $75 \%$ of sessions (PP adherence). PP analysis for girls' outcomes used the same three groups.

The effect of the intervention on girls' outcomes was examined using mixed effects logistic regressions to account for clustering. Girls' ITT and PP regressions control for age and a categorical variable indicating biological parents' presence in the home. Programme effects on caregiver outcomes were assessed using linear mixed models to account for clustering. ITT and PP models for caregivers control for caregiver gender and age. All models adjust for clustering at the programming group level, which also served as the programme session group. Additionally, ITT models for girls' outcomes adjust for clustering at the caregiver level.

Due to loss to follow-up and item non-response, outcome data are missing for approximately 10\%-20\% of girls. Pearson $\mathrm{X}^{2}$ tests and t-tests were used to assess whether missingness for girl and caregiver outcomes, respectively, was associated with treatment assignment and protocol adherence. To ensure results from the analyses above were robust to potential bias as a result of missing data, we carried out sensitivity analyses on imputed data. We used a multiple imputation approach to account for missing covariates and outcomes. 'Mi impute' was used to generate a set of five imputations in Stata, of which the average values represented a reasonable estimate of a full data set. All analyses were carried out using Stata V.14.

\section{RESULTS}

Of the 446 and 423 girls assigned to the intervention and wait-list control arms at baseline, respectively, 408 $(91.5 \%)$ and $377(89.1 \%)$ participated in endline data collection (see figure 1: Attitudes toward gender). Relocation was the most common reason for non-completion across both intervention and wait-list control arms $(\mathrm{n}=28 ; \mathrm{n}=23)$. Among the 389 and 375 caregivers assigned to the treatment and wait-list control arms at baseline, $369(94.9 \%)$ and $341(90.9 \%)$ participated in the endline survey, respectively. Relocation was also the most frequently cited reason for non-completion among caregivers. There was no statistically significant difference 
Table 1 Evaluation measures for girls and caregivers

\begin{tabular}{|c|c|c|}
\hline Outcome & Operationalisation of measure & $\begin{array}{l}\text { Possible } \\
\text { values }\end{array}$ \\
\hline \multicolumn{3}{|l|}{ Girls' outcomes } \\
\hline \multicolumn{3}{|l|}{ Primary outcome } \\
\hline Any sexual violence & $\begin{array}{l}\text { Reported experiencing forced sex, coerced sex or unwanted sexual touching } \\
\text { in the last } 12 \text { months for } 13-14 \text { year-olds; reported experiencing coerced sex } \\
\text { or unwanted sexual touching in the last } 12 \text { months for } 10-12 \text { year-olds. }\end{array}$ & $\mathrm{No}=0 ; \mathrm{Yes}=1$ \\
\hline \multicolumn{3}{|l|}{ Secondary outcomes } \\
\hline Coerced sex & $\begin{array}{l}\text { Reported having sex with someone because they threatened or pressured the } \\
\text { girl by using their influence or authority in the last } 12 \text { months. }\end{array}$ & $\mathrm{No}=0 ; \mathrm{Yes}=1$ \\
\hline Unwanted sexual touching & Being touched in a sexual way without permission in the past 12 months. & $\mathrm{No}=0 ; \mathrm{Yes}=1$ \\
\hline Forced sex (13-14 year-olds) & $\begin{array}{l}\text { Reported ever having forced sex and the most recent incident occurred within } \\
\text { the past } 12 \text { months. }\end{array}$ & $\mathrm{No}=0 ; \mathrm{Yes}=1$ \\
\hline Physical violence & Being hit or beaten in the past 12 months. & $\mathrm{No}=0 ; \mathrm{Yes}=1$ \\
\hline Emotional abuse & Someone screamed at girl loudly or aggressively in the past 12 months. & $\mathrm{No}=0 ; \mathrm{Yes}=1$ \\
\hline Neglect & Felt uncared for by the person who should provide care in the past 12 months. & $\mathrm{No}=0 ; \mathrm{Yes}=1$ \\
\hline $\begin{array}{l}\text { Child marriage (13-14 year- } \\
\text { olds) }\end{array}$ & $\begin{array}{l}\text { Reported being currently married, regardless of whether or not living with } \\
\text { spouse. }\end{array}$ & $\mathrm{No}=0 ; \mathrm{Yes}=1$ \\
\hline
\end{tabular}

Transactional sexual Reported ever having sex with someone in exchange for money, food or gifts No=0; Yes=1 exploitation and this occurred in the last 12 months.

\section{Caregiver outcomes}

Primary outcomes

$\begin{array}{ll}\begin{array}{l}\text { Attitudes towards gender } \\ \text { inequitable norms }\end{array} & \text { Composite score of agreement with } 10 \text { items regarding gender role } \\ & \text { statements. Examples include: 'It is important that sons have more education } \\ \text { than daughters'; 'Women should leave politics to men'; 'A good woman never } \\ \text { questions her husband's opinions, even if she is not sure agrees with them.' }\end{array}$

Attitudes towards physical discipline of children ${ }^{53}$
Composite score of caregivers' agreement that it is 'right' to beat a child in 11 scenarios. Examples of scenarios include: 'If the child runs away from home'; 'If the child does not want to go to work'; 'If the child steals'; 'If the child refuses to get married.'
0-10; higher

values indicate more inequitable attitudes

$0-11$; higher values indicate greater acceptance of physical discipline

\section{Parental behaviours and feelings towards their children}

\section{Parental Acceptance-} Rejection Questionnaire (PARQ) scale s4* $^{\text {PA }}$

PARQ warmth/affection subscale
Caregivers rate a series of 24 statements as they relate to their girl participating 0-96; higher in COMPASS as 'almost always true', 'sometimes true', 'rarely true' or 'almost values indicate never true'. Examples include: 'I am too busy to answer my child's questions'; greater 'I pay no attention to my child when (s)he asks for help'; 'I forget important rejection of things my child thinks I should remember'; 'I let me child know I love him/her.'

Scale derived from a subset of eight items from the full PARQ scale. Examples include: 'I say nice things about my child'; 'I make it easy for my child to confide in me'; I make my child feel wanted and needed.'

COMPASS, Creating Opportunities through Mentorship, Parental Involvement, and Safe Spaces.

*For cases where a caregiver had more than one girl participating in COMPASS, they were asked to consider their oldest daughter or girl participant. Cronbach's alpha for the overall PARQ scale was 0.75 at endline. The warmth/affection subscale was the only subscale for which alpha was greater than 0.7 ; hence, this is the only subscale presented in this analysis.

in being lost to follow-up between treatment arms for girls or caregivers.

On average, there were 24.8 girls and 21.8 caregivers in each cluster. The average age of girl participants was approximately 12 years old (see table 2 ). Because girls were matched to programme sessions by language, we observed differences in primary language spoken across treatment and wait-list control arms. Girls in the wait-list control arm also had slightly more years of schooling than those in the treatment arm. Age was the only difference 


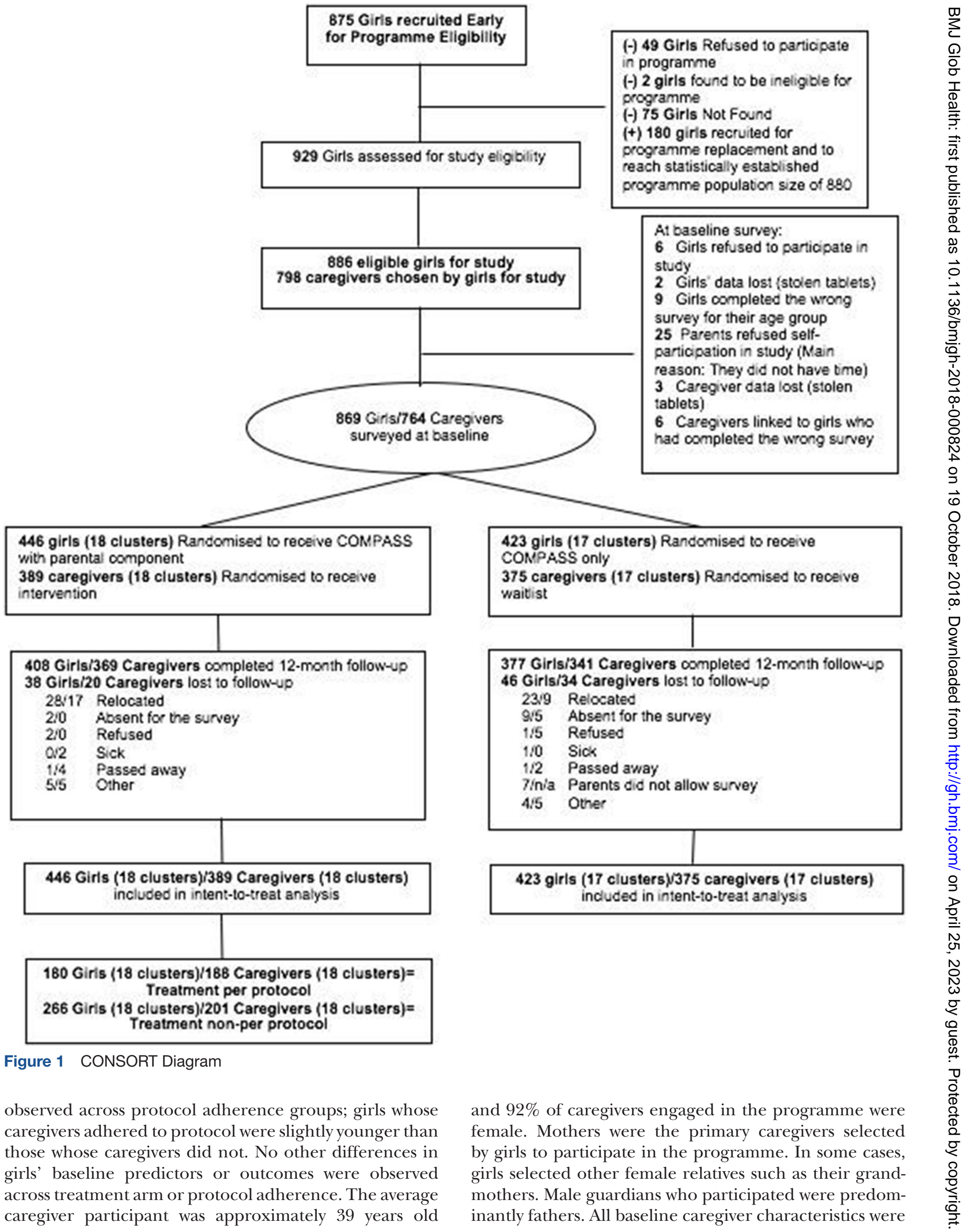


号| 00

둥유

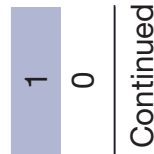

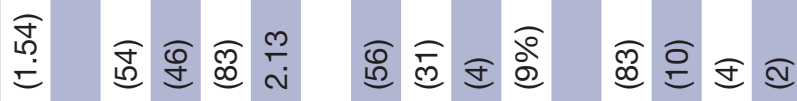

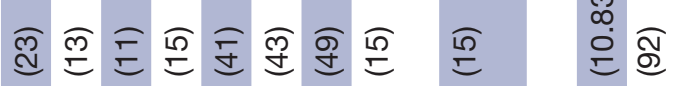

ఏ.

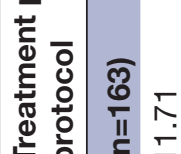

目

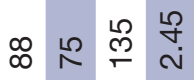

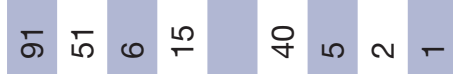

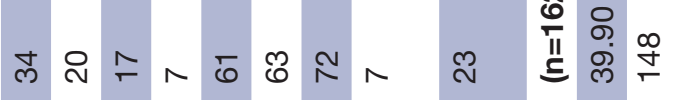

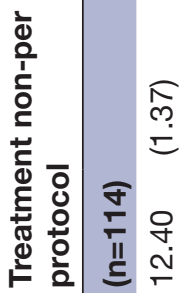

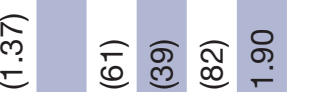

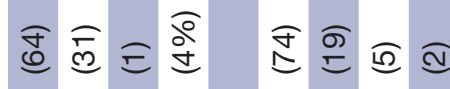

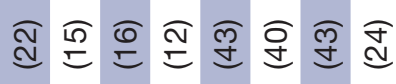

ホ̊

ต

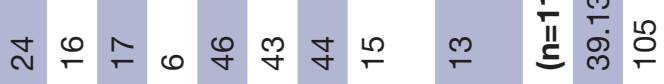

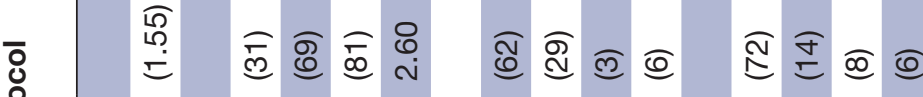

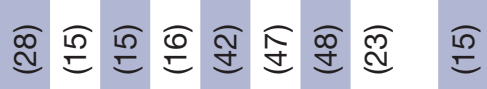

๙ุ

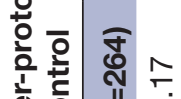

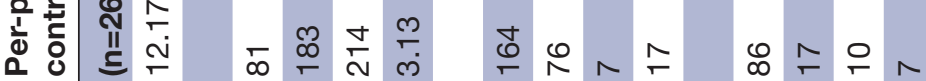

ชิ

ชิ

II N

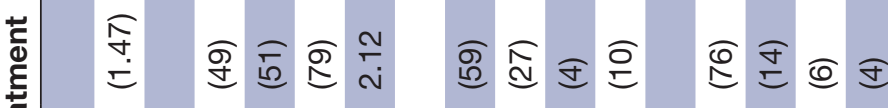

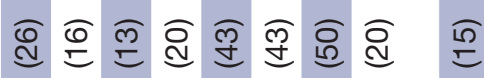

Бำ

อ

$\stackrel{1}{\frac{1}{2}}$

E I

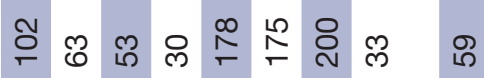

क्ष

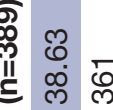

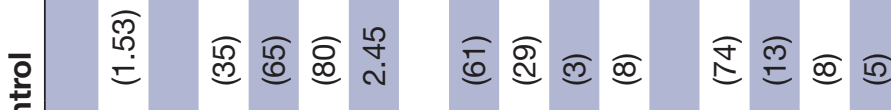

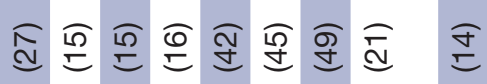

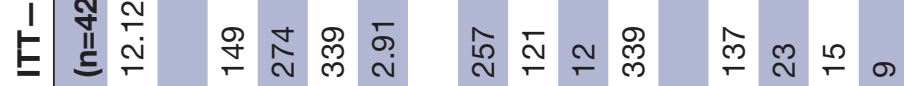

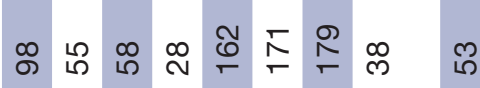

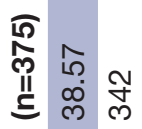

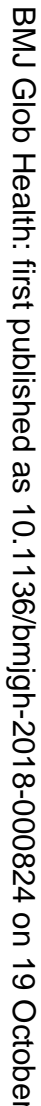

$\stackrel{\text { N }}{\stackrel{\infty}{\infty}}$

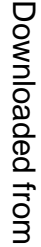
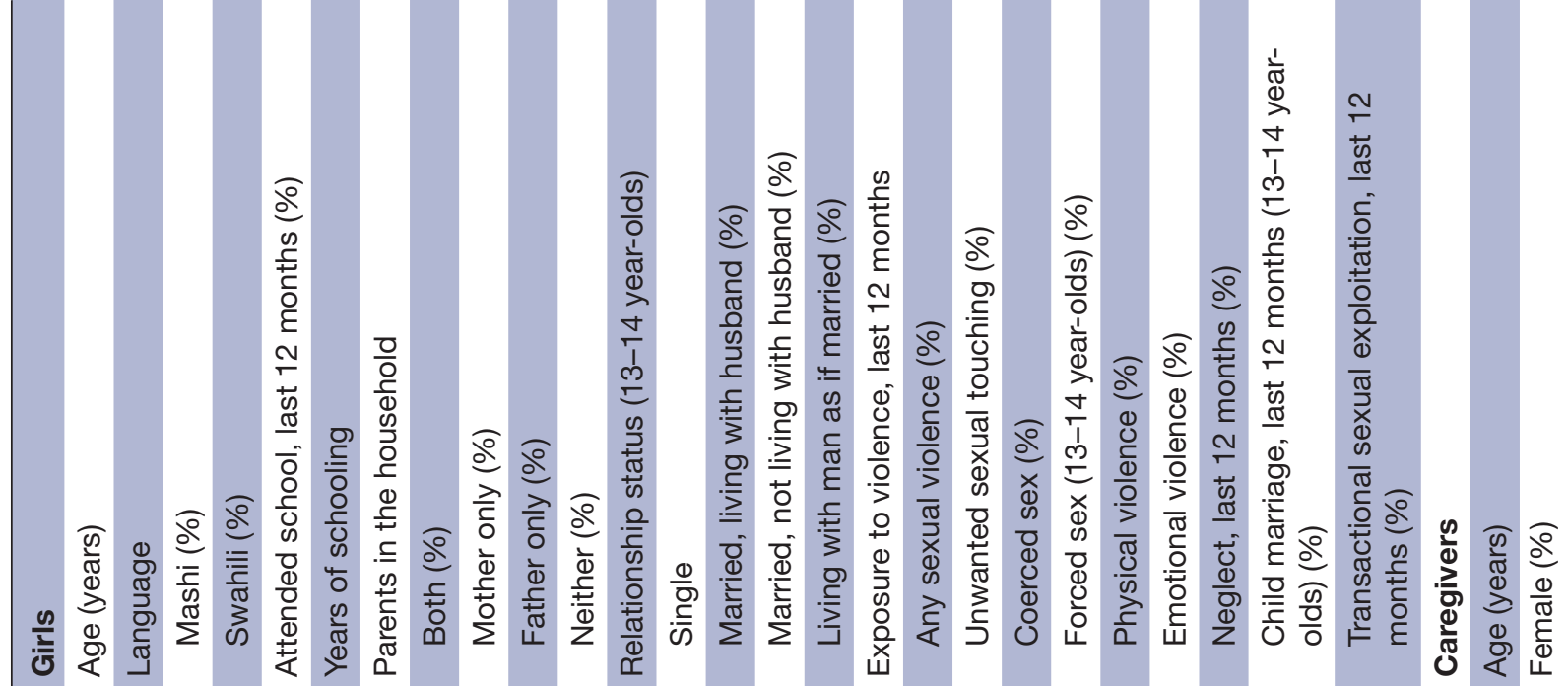


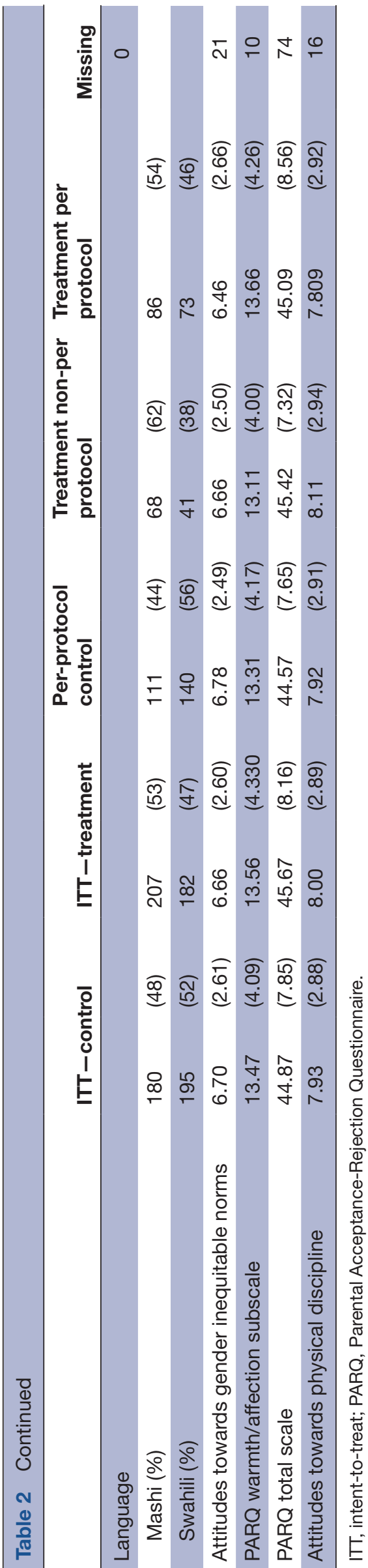

balanced across treatment and wait-list control arms, and across protocol adherence.

For both girls and caregivers' outcomes at baseline and endline, missingness was not associated with treatment status. However, having a missing value for nearly all outcomes was associated with protocol adherence; those who did not adhere to protocol were more likely to have missing outcomes, either due to loss to follow-up or item non-response.

The data show that several forms of violence decreased in the overall sample between baseline and endline (table 3 ). For example, while $27 \%$ and $26 \%$ of girls in the treatment and wait-list control arms, respectively, reported experiencing sexual in the last 12 months at baseline, these figures declined to $20 \%$ in both groups at endline. However, these declines do not appear to have been driven by the caregiver intervention. The intra-class correlation coefficient (ICC) for the primary outcome in this sample, forced sex, was 0.007 .

\section{Programme impacts}

Girls in the treatment arm were not more or less likely than those in the wait-list control to report experiencing sexual violence (adjusted $\mathrm{OR}=0.95 ; 95 \%$ CI 0.65 to 1.37 ), unwanted sexual touching, coerced sex or forced sex in the previous 12 months (table 4). Girls in the treatment arm were also just as likely to experience all forms of violence, neglect, child marriage and transactional sexual exploitation as girls in the wait-list control arm. Similarly, no programme effects were observed according to protocol adherence.

Programme impacts on caregiver outcomes are presented in table 5. After controlling for covariates, caregivers in the treatment arm had warmth/affection Parental Acceptance-Rejection Questionnaire (PARQ) subscale scores that were 1.08 points lower $(95 \%$ CI -1.79 to $0.36 ; \mathrm{p}=0.003$ ) than their wait-list control counterparts; total PARQ scales for treatment caregivers were also 2.080 lower than control caregivers $(95 \%$ CI -3.20 to $0.96 ; \mathrm{p}<0 \cdot 001)$. In other words, caregivers in the treatment arm exhibited parenting styles characterised by greater warmth and affection and lower overall rejection than those in the wait-list control arm. Similar findings hold when comparing PP adherence with the wait-list control arm. However, caregivers in the treatment arm who did not adhere to protocol were not more likely than those in the wait-list control arm to demonstrate greater warmth and affection or lower overall rejection at endline, suggesting an effect primarily driven by parents who adhered to protocol. Caregivers who participated in the intervention did not exhibit statistically significant differences in attitudes toward gender inequitable norms or acceptance of physical discipline for children, as compared with caregivers in the wait-list control group. Similar lack of impact was observed when comparing caregivers by protocol adherence. All findings are robust to sensitivity analyses on imputed data. 
Table 3 Descriptive statistics for outcomes by time period and treatment arm

\begin{tabular}{|c|c|c|c|c|c|c|c|c|c|c|}
\hline & \multicolumn{5}{|c|}{ Baseline } & \multicolumn{5}{|c|}{ Follow-up } \\
\hline & \multicolumn{2}{|c|}{ Control } & \multicolumn{2}{|c|}{ Treatment } & Missing & \multicolumn{2}{|c|}{ Control } & \multicolumn{2}{|c|}{ Treatment } & Missing \\
\hline Girls' outcomes & $(n=423)$ & & $(n=426$ & & & $(n=423)$ & & $(n=42$ & & \\
\hline \multicolumn{11}{|l|}{ Primary outcome } \\
\hline $\begin{array}{l}\text { Any form of sexual violence, last } 12 \\
\text { months (\%) }\end{array}$ & 98 & $(27)$ & 102 & $(26)$ & 114 & 69 & (20) & 74 & (20) & 155 \\
\hline \multicolumn{11}{|l|}{ Secondary outcomes } \\
\hline $\begin{array}{l}\text { Unwanted sexual touching, last } 12 \text { months } \\
\text { (\%) }\end{array}$ & 55 & $(15)$ & 63 & $(16)$ & 100 & 47 & (14) & 48 & $(14)$ & 144 \\
\hline Coerced sex, last 12 months (\%) & 58 & $(15)$ & 53 & $(13)$ & 80 & 37 & (11) & 43 & $(11)$ & 133 \\
\hline $\begin{array}{l}\text { Forced sex, last } 12 \text { months (13-14 year- } \\
\text { olds) }(\%)\end{array}$ & 28 & $(16)$ & 30 & $(20)$ & 48 & 12 & (7) & 11 & (7) & 66 \\
\hline Physical violence, last 12 months (\%) & 162 & $(42)$ & 178 & $(43)$ & 65 & 125 & (35) & 146 & $(38)$ & 134 \\
\hline Emotional violence, last 12 months (\%) & 171 & $(45)$ & 175 & $(43)$ & 79 & 130 & (38) & 146 & $(38)$ & 147 \\
\hline Neglect, last 12 months (\%) & 179 & $(49)$ & 200 & $(50)$ & 98 & 153 & $(44)$ & 172 & $(45)$ & 135 \\
\hline $\begin{array}{l}\text { Child marriage, last } 12 \text { months (13-14 } \\
\text { year-olds) (\%) }\end{array}$ & 38 & $(21)$ & 33 & $(20)$ & 27 & 28 & (18) & 29 & (19) & 68 \\
\hline $\begin{array}{l}\text { Transactional sexual exploitation, last } 12 \\
\text { months (\%) }\end{array}$ & 53 & $(14)$ & 59 & $(15)$ & 103 & 28 & (8) & 36 & $(10)$ & 150 \\
\hline Caregivers' outcomes & \multicolumn{2}{|c|}{$(n=375)$} & \multicolumn{2}{|c|}{$(n=389)$} & & \multicolumn{2}{|l|}{$(n=375)$} & \multicolumn{2}{|c|}{$(n=389)$} & \\
\hline \multicolumn{11}{|l|}{ Secondary outcomes } \\
\hline Attitudes toward gender inequitable norms & 6.70 & $(2.61)$ & 6.66 & $(2.60)$ & 21 & 5.97 & $(2.61)$ & 5.91 & $(2.71)$ & 62 \\
\hline PARQ warmth/affection subscale & 13.47 & $(4.09)$ & 13.56 & $(4.33)$ & 10 & 13.51 & $(4.08)$ & 12.45 & $(3.76)$ & 60 \\
\hline PARQ total scale & 44.87 & $(7.85)$ & 45.67 & (8.16) & 74 & 43.28 & $(7.65)$ & 41.28 & $(7.44)$ & 77 \\
\hline Attitudes toward physical discipline & 7.93 & $(2.88)$ & 8.00 & $(2.89)$ & 16 & 7.62 & $(2.83)$ & 7.55 & $(2.96)$ & 59 \\
\hline
\end{tabular}

Data are mean (SD) or number (\%). Denominator for percentages is number of respondents with a non-missing value. Some percentages do not add up to 100 because of rounding.

PARQ, Parental Acceptance-Rejection Questionnaire.

\section{DISCUSSION}

COMPASS's theory of change hypothesised that fostering supportive caregiver-girl relationships, strengthening healthy parenting styles and promoting gender equitable norms would enable caregivers to empower adolescent girls and provide a protective mechanism against sexual violence. ${ }^{41}$ This study finds no evidence that the addition of a caregiver component to this life skills programme resulted in differential exposure to any form of sexual violence, physical violence, neglect, child marriage or transactional sex for adolescent girls, beyond those of a programme that only targeted adolescent girls. However, our findings suggest at least one improvement in caregiver outcomes. As compared with caregivers in the wait-list control arm, caregivers receiving the intervention exhibited greater warmth and affection towards their children at endline, though they did not report more gender equitable attitudes, or decreased acceptance of physical discipline.

It is important to note that the realities of implementing programmes and RCTs in a humanitarian setting led to field delays in implementation and roll-out, and there was thus overlap between intervention delivery and recall periods for some outcomes of interest. This overlap ultimately limits our ability to interpret the null findings around violence exposure and the study would therefore benefit from a longer follow-up period which could capture any impact occurring post intervention completion. However, we also conducted sensitivity analyses in which we assessed programme impacts on shorter term outcomes -including self-reported exposure to forced sex in the past month and past 3 months- and found a similar lack of impact on these outcomes. Both indicators declined in the overall sample between baseline and endline; for example, incidence of forced sex in the last 3 months decreased from $15 \%$ to $7 \%$. Given that we observed a change from baseline to endline in the full sample, it is reasonable to expect to detect a difference across groups at endline if the decline had been driven by the caregiver component of the programme.

A sister evaluation examining the overall impact of the COMPASS curriculum for adolescent girls in Ethiopia also found no changes in exposure to violence for girls but did find significant changes around girls' aspirations for delaying marriage and having children, and self-reported social support systems. ${ }^{40}$ The current evaluation from the 
Table 4 Intent-to-treat and per-protocol analyses for girls' outcomes

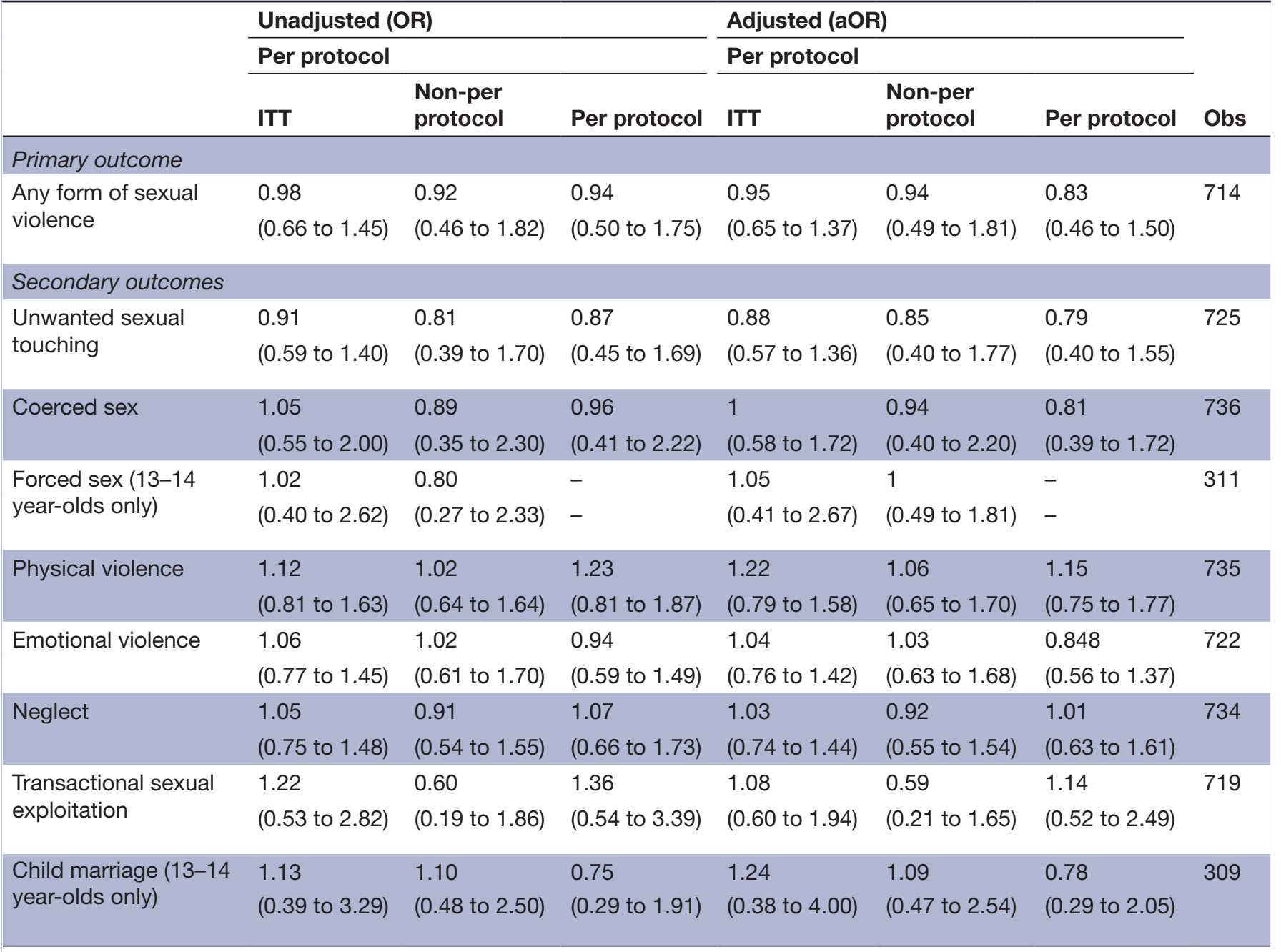

No allowance for multiplicity was made in the analyses. Adjusted models control for a four-level categorical variable indicating biological parents living in the home, and age. $95 \%$ Cls in brackets. SEs in all models are adjusted for clustering at the level of programme group; intent-to-treat (ITT) models for girls' outcomes also adjust for clustering at the level of the caregiver. ORs are statistically significant at ${ }^{*} p<0.05 ;{ }^{* *} p<0.01 ;{ }^{* \star *} p<0.001$ (there are no statistically significant ORs in this table). The reference group for the ITT columns is the full control group; the reference group for the per-protocol (PP) columns is the PP control group, that is, girls in the wait-list control arm who attended at least $75 \%$ of sessions. Differences between ORs for non-per-protocol and per-protocol adherence are not statistically significant at conventional levels. Prevalence of forced sex in blank cells is 0 and thus the OR and aOR are undefined. Obs reflects the number of observations for ITT analysis.

DRC suggests that the COMPASS caregiver curriculum was not a critical component on the pathway to change for risks to adolescent girls' well-being. The caregiver curriculum drew on best practice parenting programmes implemented in other contexts. Our findings suggest that the relevance of programme adaptations may need further attention as they relate to adolescent populations in humanitarian settings, where norms around parenting may be operating differently. Further attention to treatment fidelity may also be needed, including considering the skill level necessary for mentors and IRC staff to implement a programme like COMPASS. It is conceivable that employing more educated or better trained mentors and staff may have resulted in different outcomes. Further, the participation levels of caregivers were lower than those of girls, with programme mentors citing work, harvest, community events and sickness or pregnancy as common reasons caregivers missed sessions. These reasons suggest that caregiver curriculums and implementation strategies may need to be further adapted to better meet the needs and everyday realities of caregivers.

Several additional factors may have contributed to the limited effectiveness of the intervention. The programme's 'dosage' of one discussion group per month may have been insufficient to address deep-seated gender norms and attitudes condoning corporal punishment among caregivers. ${ }^{14}$ Further, as girls may be exposed to violence from multiple actors within the home, targeting only one caregiver per family may have resulted in a missed opportunity to holistically address girls' victimisation within the household. Structural factors outside the household also influence adolescent girls' exposure to sexual violence 
Table 5 Intent-to-treat and per-protocol analyses for caregiver outcomes

\begin{tabular}{|c|c|c|c|c|c|c|c|}
\hline & \multicolumn{3}{|c|}{ Unadjusted (beta coefficients) } & \multicolumn{3}{|c|}{ Adjusted (beta coefficients) } & \multirow[b]{3}{*}{ Obs } \\
\hline & \multicolumn{3}{|l|}{ Per protocol } & \multicolumn{3}{|l|}{ Per protocol } & \\
\hline & ITT & $\begin{array}{l}\text { Non-per } \\
\text { protocol }\end{array}$ & Per protocol & ITT & $\begin{array}{l}\text { Non-per } \\
\text { protocol }\end{array}$ & Per protocol & \\
\hline $\begin{array}{l}\text { Gender inequitable } \\
\text { roles scale }\end{array}$ & $\begin{array}{l}-0.07 \\
(-0.57 \text { to } 0.44)\end{array}$ & $\begin{array}{l}0.03 \\
(-0.70 \text { to } 0.75)\end{array}$ & $\begin{array}{l}-0.17 \\
(-0.84 \text { to } 0.50)\end{array}$ & $\begin{array}{l}-0.10 \\
(-0.61 \text { to } 0.40)\end{array}$ & $\begin{array}{l}-0.01 \\
(-0.72 \text { to } 0.70)\end{array}$ & $\begin{array}{l}-0.15 \\
(-0.80 \text { to } 0.51)\end{array}$ & 702 \\
\hline $\begin{array}{l}\text { PARQ warmth/ } \\
\text { affection subscale }\end{array}$ & $\begin{array}{l}-1.07^{\star *} \\
(-1.79 \text { to }-0.35)\end{array}$ & $\begin{array}{l}-0.87 \\
(-1.90 \text { to } 0.15)\end{array}$ & $\begin{array}{l}-1.47^{\star *} \\
(-2.40 \text { to }-0.53)\end{array}$ & $\begin{array}{l}-1.08^{\star *} \\
(-1.79 \text { to }-0.36)\end{array}$ & $\begin{array}{l}-0.9 \\
(-1.91 \text { to } 0.10)\end{array}$ & $\begin{array}{l}-1.44^{\star \star} \\
(-2.36 \text { to }-0.52)\end{array}$ & 704 \\
\hline Total PARQ scale & $\begin{array}{l}-2.00^{\star \star \star} \\
(-3.13 \text { to }-0.87)\end{array}$ & $\begin{array}{l}-1.42 \\
(-3.08 \text { to } 0.24)\end{array}$ & $\begin{array}{l}-2.18^{\star \star} \\
(-3.66 \text { to }-0.69)\end{array}$ & $\begin{array}{l}-2.08^{\star \star *} \\
(-3.20 \text { to }-0.96)\end{array}$ & $\begin{array}{l}-1.39 \\
(-3.02 \text { to } 0.25)\end{array}$ & $\begin{array}{l}-2.08^{\star *} \\
(-3.55 \text { to }-0.61)\end{array}$ & 687 \\
\hline $\begin{array}{l}\text { Acceptance of } \\
\text { physical discipline of } \\
\text { children }\end{array}$ & $\begin{array}{l}-0.07 \\
(-0.64 \text { to } 0.50)\end{array}$ & $\begin{array}{l}0.06 \\
(-0.73 \text { to } 0.84)\end{array}$ & $\begin{array}{l}-0.33 \\
(-1.06 \text { to } 0.39)\end{array}$ & $\begin{array}{l}-0.10 \\
(-0.70 \text { to } 0.49)\end{array}$ & $\begin{array}{l}0.03 \\
(-0.77 \text { to } 0.84)\end{array}$ & $\begin{array}{l}-0.3 \\
(-1.05 \text { to } 0.45)\end{array}$ & 705 \\
\hline
\end{tabular}

No allowance for multiplicity was made in the analyses. Adjusted models control for gender and age. 95\% Cls in brackets. SEs are adjusted for clustering at the level of programme group. Beta coefficients are statistically significant at ${ }^{*} p<0.05 ;{ }^{* *} p<0.01 ;{ }^{* * *} p<0.001$. The reference group for the intent-to-treat (ITT) columns is the full control group; the reference group for the per-protocol (PP) columns is the PP control group, that is, caregivers whose oldest girl participant attended at least $75 \%$ of sessions. Differences between coefficients for non-per-protocol and per-protocol adherence are not statistically significant at conventional levels. Obs reflects the number of observations for ITT analysis.

PARQ, Parental Acceptance-Rejection Questionnaire.

in these settings, and must be considered in light of the results. For example, poverty and internal displacement play a profound role in the lives of conflict-affected families and may impact perceptions of risk for both girls and caregivers. ${ }^{11}$ The struggle for survival may lead parents to expand the risks they will allow their children to take and compromise perceptions of acceptable sexual relationships for adolescents. Economic interventions, such as job training or cash transfer programmes, may alleviate some of the financial burdens families face in these settings, protecting them from having to engage in risky behaviours to ensure survival. ${ }^{43}$

While some existing evidence points to the utility of parenting programmes to reduce the utilisation of corporal punishment against children, our findings support the emerging evidence that pathways to physical violence against adolescents, and adolescent girls in particular, may be more nuanced. ${ }^{346}$ While our study shows that caregiver discussion groups have the potential to positively transform parenting styles, we do not observe impacts further along the causal pathway in regard to reducing girls' exposure to any measured form of violence. If positive shifts in parenting styles are hypothesised to be a very early indicator of change, programme evaluations may need additional follow-ups to assess changes in outcomes farther out from the programme cycle. Additionally, the COMPASS curriculum was condensed to fit within an emergency context, but it may need to be implemented over a longer period to affect sustainable changes. Given the nascent nature of this type of programming, adolescent interventions that include a parenting component in conflict settings may benefit from additional implementation analysis to better understand the mechanisms of curriculum delivery that compel caregivers to take action, as well as identify potential limitations to their doing so.

Future programmes may also need to engage other influential people in girls' lives, and consider social norms on gender equity, in order to affect change. While caregivers may be influential in setting normative standards of behaviour for adolescents, caregivers are themselves influenced by social norms, which may set gendered parameters on individual authority within both the household and the community. ${ }^{44}$ For example, although the programme was open to male caregivers, the vast majority of caregivers chosen by adolescents, who were asked to identify a 'safe caregiver' to participate in the caregiver curriculum, were female. In a patriarchal society that normalises certain forms of violence, opportunities for women to influence community-level social norms as well as decision-making about child-rearing practices in the home may both be limited and put women at risk of violence. ${ }^{45}{ }^{46}$ Thus, girls' perceptions of a safe caregiver may not reflect that caregiver's influence in establishing a supportive home environment for adolescent girls. Further, evidence shows there is often little communication between girls and caregivers on these sensitive issues, suggesting caregivers may have limited opportunities to share what they learnt through the programme. ${ }^{47}$

Working with influential community members, such as religious leaders or community elders, on social norms regarding gender equity may have a more powerful and sustainable impact on the community's perception of these issues. Interventions should also work specifically with men and boys to reduce violence against adolescent girls, especially since intimate partners may perpetrate a substantial share of violence against adolescent girls in 
these settings. ${ }^{11}$ Furthermore, witnessing spousal abuse or IPV in the home as a child is a known risk factor for girls' later exposure to IPV. ${ }^{19} 48$ Targeting fathers as decision-makers and potential perpetrators of IPV in the home may therefore reduce girls' likelihood of experiencing violence in adolescence and later in life. Additionally, findings on violence indicate an urgent need for effective strategies to prevent violence against adolescent girls and provide support to adolescent survivors of violence, especially if caregivers may hold attitudes that blame girls for violence exposure. ${ }^{49}$ Violence interventions in humanitarian settings often focus on tertiary interventions, such as survivor support services; greater attention should be paid to primary prevention. ${ }^{50}$

This study has a few limitations stemming from the difficulties of implementing an RCT in a conflict setting. First, while randomising sisters to the same group minimised within-household contamination, existence of both treatment and control groups in some villages may have facilitated within-village contamination if caregivers in the intervention shared information from programme sessions with non-intervention caregivers; such within-village contamination would bias the results towards the null. It is also important to note that all outcomes of interest are subject to disclosure bias as they are self-reported. ACASI was used to limit social desirability bias in soliciting sensitive information from adolescents, and has demonstrated effectiveness in this regard among adolescents. ${ }^{51}$ Finally, while findings from this study may be generalisable for conflict-affected girls in similar communities in South Kivu, we cannot necessarily assume that the findings would extend to other populations in the region.

\section{CONCLUSION}

Despite global calls to prioritise adolescent health and well-being, there remains a dearth of evidence on preventing violence and promoting well-being for adolescent girls in emergencies. As caregivers play an instrumental and proximal role in adolescent girls' lives, the types of emotional, parental and social support they provide may plausibly shape girls' susceptibility to violence through a number of pathways. While this study finds that a caregiver programme improved some parenting behaviours, it finds no evidence that the addition of this programming to a girl's life skills intervention was associated with decreased exposure to violence for girls. Further research is needed to understand these pathways and to inform efficacious interventions to engage caregivers in keeping adolescent girls safe from violence. We recommend future efforts to include longer term evaluation follow-up and expansion of programme targeting to also include men (e.g., fathers, brothers, boyfriends and husbands) and other key figures in the community to support the safety and well-being of women and girls in humanitarian contexts.
Author affiliations

${ }^{1}$ George Warren Brown School of Social Work, Washington University in Saint Louis, St. Louis, Missouri, USA

${ }^{2}$ Department of Population and Family Health, Columbia University Mailman School of Public Health, New York City, New York, USA

${ }^{3}$ International Rescue Committee, New York City, New York, USA

${ }^{4}$ Independent Consultant, Paris, France

Contributors LS is the principal investigator and led the manuscript development. IS led the data analysis and supported the manuscript development. KA supported the data analysis, interpretation and manuscript development. KLF, MM and CFD supported the conceptual development and study implementation. DR and TB managed the tool adaptation and oversight of data collection. All authors reviewed and approved the final manuscript prior to submission. All authors had full access to all of the data (including statistical reports and tables) in the study and can take responsibility for the integrity of the data and the accuracy of the data analysis. The corresponding author had full access to all the data in the study and had final responsibility for the decision to submit for publication.

Funding The study was funded by the UK Department for International Development (grant number 40080602).

Disclaimer The funders played no role in the study design; in the collection, analysis and interpretation of data; in the writing of the report; and in the decision to submit the article for publication. The sponsor of the study had no role in study design, data collection, data analysis, data interpretation, or writing of the report.

Competing interests None declared.

Patient consent Not required.

Ethics approval The Columbia University Medical Center Institutional Review Board (protocol number AAAP6855); Ministry of Gender in South Kivu, DRC

Provenance and peer review Not commissioned; externally peer reviewed.

Data sharing statement Due to legal restrictions, data cannot be made publicly available. Data are owned by the International Rescue Committee (IRC). For more information about IRC data, please visit the following URL: http://www.whatworks. co.za/about/about-what-works. For data related inquiries please contact Kathryn Falb (Kathryn.Falb@rescue.org).

Open access This is an open access article distributed in accordance with the Creative Commons Attribution Non Commercial (CC BY-NC 4.0) license, which permits others to distribute, remix, adapt, build upon this work non-commercially, and license their derivative works on different terms, provided the original work is properly cited, appropriate credit is given, any changes made indicated, and the use is non-commercial. See: http://creativecommons.org/licenses/by-nc/4.0

\section{REFERENCES}

1. Mikton C, Butchart A. Child maltreatment prevention: a systematic review of reviews. Bull World Health Organ 2009;87:353-61.

2. WHO. INSPIRE: seven strategies for ending violence against children. Geneva: WHO, 2016.

3. Sim A, Annan J, Puffer E. Building happy families: impact evaluation of a parenting and family skills intervention for migrant and displaced burmese families in thailand. New York: International Rescue Committee, 2014.

4. Sim A, Puffer E, Green E. Parents make the difference: findings from a randomized impact evaluation of a parenting program in rural Liberia. New York: International rescue committee, 2014.

5. Ashburn K, Kerner B, Ojamuge D. Evaluation of the responsible, engaged, and loving (real) fathers initiative on physical child punishment and intimate partner violence in northern uganda. Prevention Science 2016:1-11.

6. Puffer ES, Annan J, Sim AL, et al. The impact of a family skills training intervention among burmese migrant families in thailand: a randomized controlled trial. PLoS One 2017;12:e0172611.

7. UNICEF. Ending violence against children: six strategies for action. New York: UNICEF, 2014b.

8. Knerr W, Gardner F, Cluver L. Improving positive parenting skills and reducing harsh and abusive parenting in low- and middle-income countries: a systematic review. Prev Sci 2013;14:352-63.

9. Cluver LD, Lachman JM, Ward CL. Development of a parenting support program to prevent abuse of adolescents in South Africa findings from a pilot pre-post study. Res Soc Work Pract 2016;27. 
10. Lundgren R, Amin A. Addressing intimate partner violence and sexual violence among adolescents: emerging evidence of effectiveness. Journal of Adolescent Health 2015;56:S42-S50.

11. Stark L, Asghar K, Yu G, et al. Prevalence and associated risk factors of violence against conflict-affected female adolescents: a multi-country, cross-sectional study. J Glob Health 2017;7:1.

12. Raj $A$. When the mother is a child: the impact of child marriage on the health and human rights of girls. Arch Dis Child 2010;95:931-5.

13. UNICEF. A statistical snapshot of violence against adolescent girls. New York: UNICEF, 2014a.

14. Falb KL, Asghar K, Laird B, et al. Caregiver parenting and gender attitudes: associations with violence against adolescent girls in South Kivu, democratic republic of congo. Child Abuse Negl 2017;69:278-84.

15. Heise L. Preventing intimate partner violence. Oxford textbook of violence prevention. In: Donnelly P, Ward C, Epidemiology, evidence, and policy. eds. Oxford: Oxford University Press, 2015:185-92.

16. Kast NR, Eisenberg ME, Sieving RE. The role of parent communication and connectedness in dating violence victimization among latino adolescents. J Interpers Violence 2016;31:1932-55.

17. Shlafer RJ, McMorris BJ, Sieving RE, et al. The impact of family and peer protective factors on girls' violence perpetration and victimization. J Adolesc Health 2013:52:365-71.

18. Ohene SA, Ireland M, McNeely C, et al. Parental expectations, physical punishment, and violence among adolescents who score positive on a psychosocial screening test in primary care. Pediatrics 2006;117:441-7.

19. Widom CS, Czaja S, Dutton MA. Child abuse and neglect and intimate partner violence victimization and perpetration: a prospective investigation. Child Abuse Negl 2014;38:650-63.

20. Heise LL. Violence against women: an integrated, ecological framework. Violence Against Women 1998;4:262.

21. Mann JR, Takyi BK. Autonomy, dependence or culture: Examining the impact of resources and socio-cultural processes on attitudes towards intimate partner violence in Ghana, Africa. J Fam Violence 2009;24:323-35

22. Yount KM, Higgins EM, VanderEnde KE, et al. Men's perpetration of intimate partner violence in Vietnam. Men Masc 2016;19:64-84.

23. Khawaja M, Linos N, El-Roueiheb Z. Attitudes of men and women towards wife beating: Findings from palestinian refugee camps in Jordan. J Fam Violence 2008;23:211-8.

24. van de Gaag N. In double jeopardy: adolescent girls and disasters. In: Because i am a girl: State of the World's Girls 2013. Surrey, UK: Plan International, 2013.

25. Murphy KM, Rodrigues K, Costigan J, et al. Raising children in conflict: an integrative model of parenting in war. Peace and Conflict: Journal of Peace Psychology. 2017;23:46-57.

26. Catani C, Schauer E, Neuner F. Beyond individual war trauma: Domestic violence against children in afghanistan and Sri Lanka. J Marital Fam Ther 2008;34:165-76.

27. Spencer D. CARE International UK. "To protect her honour": Child marriage in emergencies - the fatal confusion between protecting girls and sexual violence, 2015

28. UNHCR and Save the Children-UK. The experience of refugee children in Guinea, Liberia and Sierra Leone: sexual violence \& exploitation, 2002

29. WHO. WHO multi-country study on women's health and domestic violence against women: summary report of initial results on prevalence, health outcomes and women's responses. Geneva: WHO, 2005

30. Erulkar A, Ferede A. Social exclusion and early or unwanted sexual initiation among poor urban females in Ethiopia. Int Perspect Sex Reprod Health 2009;35:186-93.

31. Lautze S, Raven-Roberts A. Violence and complex humanitarian emergencies: implications for livelihoods models. Disasters 2006;30:383-401.

32. Decker MR, Latimore AD, Yasutake S, et al. Gender-based violence against adolescent and young adult women in low- and middleincome countries. J Adolesc Health 2015;56:188-96.
33. Mels C, Derluyn I, Broekaert E, et al. Screening for traumatic exposure and posttraumatic stress symptoms in adolescents in the war-affected eastern Democratic Republic of Congo. Arch Pediatr Adolesc Med 2009;163:525-30.

34. Exner-Cortens D, Eckenrode J, Rothman E. Longitudinal associations between teen dating violence victimization and adverse health outcomes. Pediatrics 2013;131:71-8.

35. Nöthling J, Suliman S, Martin L, et al. Differences in abuse, neglect, and exposure to community violence in adolescents with and without PTSD and depression. J Interpers Violence. 2016:088626051667494

36. Koenig MA, Zablotska I, Lutalo T, et al. Coerced first intercourse and reproductive health among adolescent women in Rakai, Uganda. Int Fam Plan Perspect 2004:30:156-63.

37. Thornberry TP, Ireland TO, Smith CA. The importance of timing: the varying impact of childhood and adolescent maltreatment on multiple problem outcomes. Dev Psychopathol 2001;13:957-79.

38. Olofsson N, Lindqvist K, Shaw BA, et al. Long-term health consequences of violence exposure in adolescence: a 26-year prospective study. BMC Public Health 2012;12:411.

39. Johnson MK, Crosnoe R, Elder GH. insights on adolescence from a life course Perspective. J Res Adolesc 2011;21:273-80.

40. Stark L, Asghar K. Preventing violence against refugee adolescent girls. Findings from a Randomized Controlled Trial in Ethiopia, 2016.

41. Falb KL, Tanner S, Ward L, et al. Creating opportunities through mentorship, parental involvement, and safe spaces (COMPASS) program: multi-country study protocol to protect girls from violence in humanitarian settings. BMC Public Health 2016;16:231.

42. Falb K, Tanner S, Asghar K, et al. Implementation of audiocomputer assisted self-Interview (ACASI) among adolescent girls in humanitarian settings: feasibility, acceptability, and lessons learned. Confl Health 2016;10:32.

43. ODI. Doing Cash Differently. How Cash transfers can transform humanitarian aid. London, 2015.

44. Biddle BJ, Bank BJ, Marlin MM. Parental and Peer Influence on adolescents. Social Forces 1980;58:1057-79.

45. Freedman J. Explaining sexual violence and gender inequalities in the DRC. Peace Review 2011;23:170-5.

46. Kelly J, Kabanga J, Cragin W, et al. 'If your husband doesn't humiliate you, other people won't': gendered attitudes towards sexual violence in eastern Democratic Republic of Congo. Glob Public Health 2012;7:285-98.

47. Sommer M, Munoz-Laboy M, Salamea E. How narratives of fear shape girls' participation in community life in two humanitarian contexts. Violence Against Women 2017.

48. Abramsky T, Watts $\mathrm{CH}$, Garcia-Moreno $\mathrm{C}$, et al. What factors are associated with recent intimate partner violence? findings from the WHO multi-country study on women's health and domestic violence. BMC Public Health 2011;11:109.

49. Sommer M, Muñoz-Laboy M, Williams A, et al. How gender norms are reinforced through violence against adolescent girls in two conflict-affected populations. Child Abuse Negl 2018;79:154-63.

50. Women's Refugee Commission. Minimum initial service package (MISP) for reproductive health in crisis situations: a distance learning module, 2006.

51. Mensch BS, Hewett PC, Gregory R, et al. Sexual behavior and STI/HIV status among adolescents in rural Malawi: an evaluation of the effect of interview mode on reporting. Stud Fam Plann 2008;39:321-34.

52. Nanda G. Compendium of gender scales. Washington, DC, 2011 FHI 360/C-Change.

53. Ruiz-Casares M. Save the Children. Child protection knowledge, attitudes, and practices in central and Western Liberia, 2011.

54. Rohner R, Saavedra J, Granum E. Development and validation of the parental acceptance rejection questionnaire: test manual. JSAS catalogue of selected documents in psychology 1978;8:7-8. 